\title{
Therapeutic applications of transcription factor decoy oligonucleotides
}

\author{
Michael J. Mann ${ }^{1}$ and Victor J. Dzau ${ }^{2}$ \\ ${ }^{1}$ Department of Surgery, and \\ 2Department of Medicine, Brigham and Women's Hospital, Boston, Massachusetts, USA
}

Address correspondence to: Victor J. Dzau, Tower 1, Office of the Chairman, Department of Medicine, Brigham and Women's Hospital, Harvard Medical School, 75 Francis Street, Boston, Massachusetts 02115, USA

Phone: (617) 732-6340; Fax: (617) 732-6439; E-mail: vdzau@partners.org.

As alterations in gene expression have become a better understood component of normal development and disease pathogenesis, transcription factors and other regulators of gene expression have become an increasingly attractive target for potential therapeutic intervention. Transcription factors are generally nuclear proteins that play a critical role in gene regulation and can exert either a positive or negative effect on gene expression. These regulatory proteins bind specific sequences found in the promoter regions of their target genes. These binding sequences are generally $6-10 \mathrm{bp}$ in length and are occasionally found in multiple iterations.

Although this protein-DNA interaction is quite sequence-specific, the binding sites for a single transcription factor may vary by several base pairs when found in the promoter regions of different genes; in this case, a common motif, or consensus binding site, can be described. Often a number of different transcription factor binding sites are located in the regions both upstream and downstream from a given transcription initiation site. The binding of these different factors, and the subsequent interactions of these proteins with each other, as well as with RNA polymerases or their cofactors, yield a complex set of conditions that determines the relative transcriptional activity at different times and under varying conditions in different cell types.

The transcription factor decoy approach

Because transcription factors can recognize their relatively short binding sequences even in the absence of surrounding genomic DNA, short radiolabeled oligodeoxynucleotides (ODNs) bearing consensus binding sites can serve as probes in electrophoretic mobility shift assays, which identify and quantify transcription factor binding activity in nuclear extracts. More recently, ODNs bearing the consensus binding sequence of a specific transcription factor have been explored as tools for manipulating gene expression in living cells. This strategy involves the intracellular delivery of such "decoy" ODNs, which are then recognized and bound by the target factor. Occupation of the transcription fac- tor's DNA-binding site by the decoy renders the protein incapable of subsequently binding to the promoter regions of target genes. Bielinska and coworkers first described the use of such decoys as a tool for investigating the role of transcription factor activity in cell culture systems (1). Similar decoys can also be devised as therapeutic agents, either to inhibit the expression of genes that are transactivated by the factor in question, or to upregulate genes that are transcriptionally suppressed by the binding of a factor (Figure 1).

The use of decoy ODNs for the therapeutic manipulation of gene expression was first described by our laboratory in 1995. Morishita et al. reported the treatment of rat carotid arteries at the time of balloon injury with ODNs bearing the consensus binding site for the E2F family of transcription factors (2). Although as many as six E2F isoforms are known to play differing roles in cell cycle progression and cell growth (3), release of the predominant E2F-1 isoform at a critical point in the late G1 phase of the cell cycle coordinately upregulates expression of multiple genes that help speed the cell through DNA synthesis and mitosis. We found that a decoy specific to E2F-1 prevented this upregulation and blocked smooth muscle proliferation and neointimal hyperplasia in injured vessels (2). In addition to this initial in vivo application, our group has used a transcription factor decoy to block a negative regulatory element in the promoter of the Renin gene in the mouse submandibular gland, demonstrating that decoys can be used to increase as well as to suppress gene activity in vivo $(4,5)$.

\section{From bench to bedside}

The successful blockade of multiple cell cycle genes via the delivery of a single decoy ODN directed against E2F, as well as the subsequent inhibition of neointimal hyperplasia observed in a model of arterial injury, prompted us to consider the application of this strategy to human therapy. We selected for our first therapeutic target human bypass vein graft failure, a process characterized by neointimal hyperplasia and accelerated atherosclerosis. Despite long-term failure rates that approach $50 \%$, 


\begin{tabular}{|c|}
\hline Nucleic acid therapeutics \\
\hline Bruce A. Sullenger, Series Editor
\end{tabular}

a

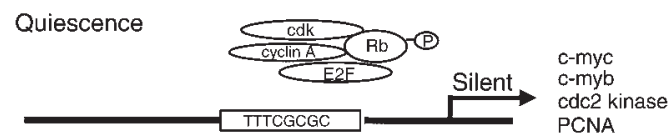

b

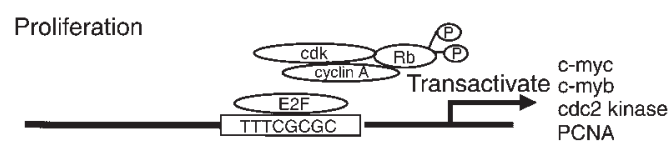

c

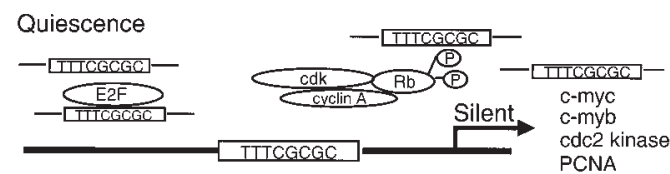

Figure 1

The decoy oligonucleotide approach to block the function of the transcription factor E2F. In quiescent cells (a), the factor is sequestered in a protein complex. During cell cycle progression (b), the complex is phosphorylated and free E2F is released. The factor binds to its consensus binding sequence in the promoter regions of multiple cell cycle regulatory genes. The introduction into the nucleus of decoy oligonucleotides that bear the consensus binding sequence (c) prevents interaction of the factor with its promoter targets, thus inhibiting the upregulation of cell cycle genes and blocking proliferation.

autologous vein grafts remain the most widely used conduit for surgical revascularization in roughly 600,000 patients annually in the US who suffer from occlusive disease of the coronary or lower extremity circulations. Failures, which can lead to reoperation, amputation, or heart attack, can be traced to the development of a neointimal layer during the postoperative remodeling of the initially thin-walled vein in its new high-pressure arterial environment (6). Although such neointimal thickening relieves tangential wall stress in the bypass vessel (7), the expression of cytokines, adhesion molecules, and chemoattractants by the activated neointimal smooth muscle cells is associated with dysfunction of the overlying endothelium, leukocyte invasion of the vessel wall, and the development of an aggressive, accelerated form of atherosclerosis (Figure 2) $(6,8)$.

We hypothesized that E2F decoy-mediated arrest of cell cycle progression during the early postoperative period would block this neointimal hyperplastic response to the acute injuries associated with grafting. Furthermore, this intervention would allow a more desirable hypertrophic response in the medial layer, which would help the vessel accommodate to the chronic hemodynamic stress of the arterial circulation. Indeed, a single, intraoperative transfection of rabbit vein grafts with E2F decoy provided a long-term inhibition of neointimal hyperplasia and led to a shift toward medial hypertrophy, which succeeded in reducing wall stress to near-arterial levels (9). Furthermore, the inhibition of neointimal hyperplasia helped preserve vein graft endothelial function and prevented diet-induced graft atherosclerosis for at least 6 months after operation $(9,10)$.
On the strength of these preclinical data, we initiated a clinical program of prospective, double-blind, randomized evaluation of E2F decoy transfection of human bypass vein grafts (11). The initial study involved a small cohort of infrainguinal bypass patients, including a large proportion of individuals at high risk for neointimal disease and graft failure. Because of the anatomic location of the vein grafts, we were able to use ultrasound (12) to monitor them for critical lesion formation in a reliable and noninvasive manner. To carry out ex vivo E2F decoy transfection, we exposed the grafts to a "naked" ODN solution under a nondistending pressure of $300 \mathrm{mmHg}$, in a process that required only 10-15 minutes and could be carried out while the patient's leg was prepared for grafting. Little, if any, systemic exposure to the E2F decoy occurred during this procedure. Small specimens of transfected vein were collected for laboratory evaluation, which revealed an $89 \%$ transfection efficiency and a sequence-specific inhibition both of target cell cycle mRNA expression and of vascular cell replication in an organ culture assay. This ex vivo, pressure-mediated E2F decoy transfection of human vein grafts did not influence either postoperative complication rates or blood counts and chemistries.

Despite the small size of this preliminary, single-center trial, the choice of a high-risk cohort allowed us to compare the occurrence of primary graft failure (graft occlusion or a need for invasive graft revision) between patients who did and those who did not receive the E2F decoy treatment. We found that, whereas failures occurred among the untreated cohort throughout the 12-month follow up period, no failures were observed
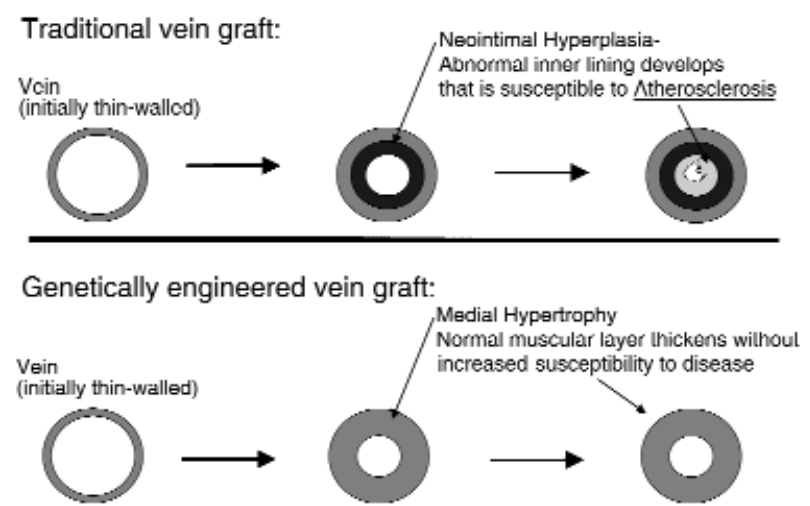

Figure 2

Vein grafts are initially thin-walled vessels that must undergo wall thickening to resist increased stress in the arterial circulation. The neointimal hyperplasia that produces this thickening, however, involves the proliferation of activated smooth muscle cells that create a substrate for accelerated atherosclerosis and subsequent graft occlusion. Blocking neointimal hyperplasia, as we have done using an E2F decoy oligonucleotide, induces an adaptive hypertrophic thickening of the medial layer of the vessel, yielding hemodynamic stability without increased susceptibility to atherosclerotic disease. 
among the E2F decoy-treated grafts beyond the first 6 months, suggesting that the adaptive remodeling seen in preclinical experiments also occurred in the human grafts (Figure 3). Larger-scale clinical trials will be necessary to verify the results, but this work, one of the first attempts to apply an ex vivo gene-based strategy to treat a common cardiovascular disorder, offers the first evidence that human gene expression can be modulated for therapeutic purposes using a transcription factor decoy.

New therapeutic targets

A growing number of transcription factor proteins have been identified that regulate changes in gene expression during the pathogenesis of a wide range of disorders. The decoy approach therefore represents a potential avenue for broad clinical application, a sampling of which is outlined in Table 1. A number of groups have adopted the transcription factor decoy approach and have begun to establish proof of principle in some of these applications.

$\mathrm{NF}-\kappa \mathrm{B}$ has been identified as a critical mediator of the cellular response to oxidative stress (13). The distribution of binding sites for NF- $\mathrm{KB}$ implies that this transcription factor regulates a wide variety of genes, particularly those for interleukins and for adhesion molecules that play critical roles in inflammation (14). Khaled et al. (15) report that an NF- $\kappa B$ decoy ODN can reduce IgG and anti-DNA antibody production in cul- tured splenocytes. This treatment also blocks expression of TNF- $\alpha$, IL-2, and IL- 6 for up to 72 hours, suggesting that it might be useful in treating autoimmune diseases. Griesenbach and colleagues have tested a similar anti-inflammatory therapy for cystic fibrosis patients, in which TNF- $\alpha$-induced secretion of IL- 8 is inhibited by the NF- $\mathrm{kB}$ decoy in a cystic fibrosis epithelial cell line (16). Their cell culture data are further supported by an in vivo investigation of NF- $\kappa \mathrm{B}$ decoy ODN injection into the hind ankle joints in a rat model of rheumatoid arthritis (17). The decoy, encapsulated in a fusigenic liposome to improve cellular uptake, decreased not only IL- 1 and TNF- $\alpha$ expression in the joints, but also paw swelling and joint destruction over a 28-day period. Another group examined the role of $\mathrm{NF}-\kappa \mathrm{B}$ transactivation in tumor-induced cachexia in mice and found that intratumoral injection of NF- $\kappa B$ decoy ODN into colonic adenocarcinoma blunted drops in food intake, body weight, and muscle mass, although this treatment did not influence the growth of the tumor itself (18).

NF- $\kappa \mathrm{B}$-mediated inflammation also influences development of a number of cardiovascular disorders. In particular, NF- $\mathrm{KB}$ activation in vascular endothelial cells is believed to upregulate cytokines and adhesion molecules during the response to ischemia/reperfusion and to contribute to subsequent myocardial dysfunction and necrosis. Thus, cultured endothelial cells

Table 1

Transcription factors as potential therapeutic targets for gene manipulation

\begin{tabular}{|c|c|c|}
\hline Transcription factor & Cellular process & Potential therapeutic application \\
\hline $\mathrm{E} 2 \mathrm{~F}$ & cell proliferation & $\begin{array}{l}\text { neointimal hyperplasia (vein graft disease, restenosis), } \\
\text { neoplasia, glomerulonephritis, angiogenesis, inflammation }\end{array}$ \\
\hline $\mathrm{NF}-\kappa \mathrm{B}$ & $\begin{array}{c}\text { cytokine expression, leukocyte adhesion molecule } \\
\text { expression, oxidant stress response, cAMP } \\
\text { and protein kinase } C \text { activation, Ig expression }\end{array}$ & $\begin{array}{l}\text { inflammation, immune response, transplant rejection, } \\
\text { ischemia reperfusion injury, glomerulonephritis }\end{array}$ \\
\hline CREB & cAMP response & cAMP activated events, neoplasia \\
\hline MEF-2 & cardiac myocyte differentiation and hypertrophy & cardiomyopathy, heart failure \\
\hline CarG box & cardiac myocyte differentiation & cardiomyopathy, heart failure \\
\hline $\operatorname{tax}$ & viral replication & HTLV infection \\
\hline VP16 & viral replication & Herpes virus infection \\
\hline GRE/HRE/MRE & glucocorticoid/mineralocorticoid-induced events & $\begin{array}{l}\text { steroid hormone-mediated processes } \\
\text { (breast cancer, prostate cancer, etc.) }\end{array}$ \\
\hline Heat shock RE & heat shock response & cellular stress (ischemia, hypoxia) \\
\hline SRE & growth factor responses & cell proliferation/differentiation \\
\hline AP-2 & cAMP and protein kinase response, retinoic acid response & cell proliferation \\
\hline sterol response element & modulation of LDL receptor expression & hypercholesterolemia \\
\hline TGF- $\beta$ responsive element & TGF- $\beta$-induced cellular processes & $\begin{array}{l}\text { cell growth and differentiation, migration, angiogenesis, } \\
\text { neointimal hyperplasia, matrix generation, apoptosis }\end{array}$ \\
\hline HIF-1 & response to hypoxia, angiogenesis & antiangiogenesis (neoplasia) \\
\hline
\end{tabular}



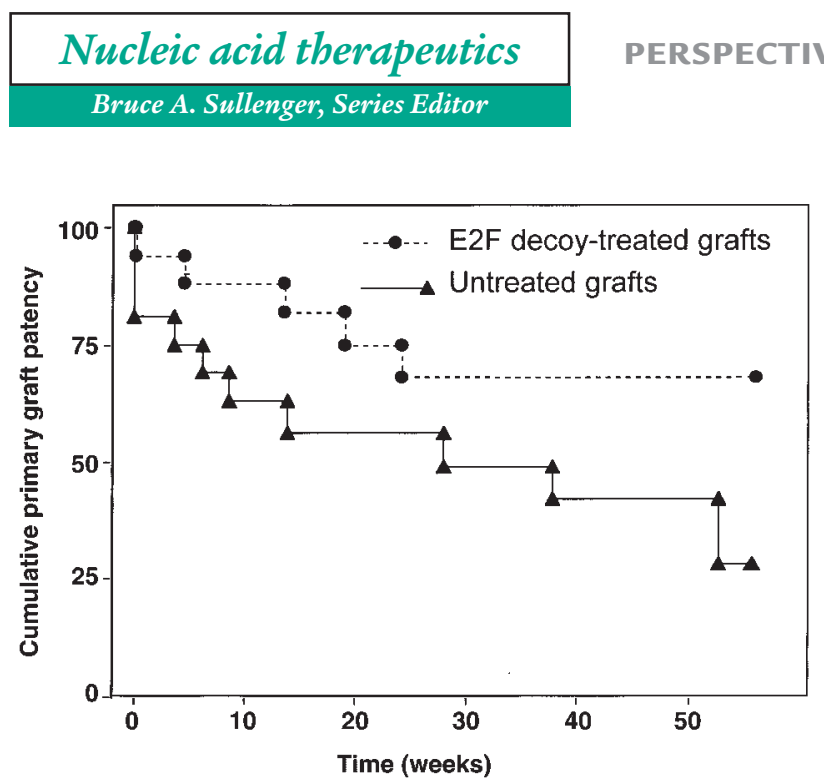

Figure 3

Primary vein graft patency with or without intraoperative treatment of the grafts with E2F decoy oligonucleotides. In a small cohort of patients at high risk for primary graft failure, primary patency at 12 months was improved with E2F decoy blockade of cell cycle gene expression and neointimal hyperplasia $(P=0.04)$. The absence of ongoing failures beyond the first 6 months in the treated group may suggest a long-term stabilization of graft wall architecture via this one-time, intraoperative gene manipulation approach. (From ref. 11).

treated with the NF- $\mathrm{\kappa B}$ decoy are insensitive to specific inhibition of TNF- $\alpha$-induced upregulation of IL- 6 and intercellular adhesion molecule-1 (19), and coronary perfusion with liposomes containing the decoy improves coronary blood flow and left ventricular pressure after ischemia/reperfusion injury (20). In a similar study, Morishita et al. (21) showed that in vivo decoy therapy reduced myocardial infarct size in a rat model of acute coronary occlusion.

Decoy ODNs also offer a means to specifically inhibit other transcription factors in living cells, both for basic research into the molecular pathways involving these factors (22-27) and for novel drug development. Promising therapeutics based on this approach include inhibitors of Stat6, which may be useful in reducing IL-4-induced proliferation of Th cells in allergic diseases (28), and of the cAMP-response element-binding protein (CREB) in tumor cells (29). CREB transcription factor decoys have yielded a particularly intriguing effect on the in vitro and in vivo growth of a number of tumor cell lines, in that growth inhibition appears to be tumor-specific and has not been observed in any of the noncancerous cells tested. Although the basis for this cell specificity has not yet been elucidated, CREB decoys have been associated with sequence-specific stabilization of the p53 tumor suppressor in breast cancer cells (30).

Limitations and challenges

The multiplicity of transcription factors that regulate a given gene and the multiplicity of target genes that are under control of a single transcription factor represent important limitations to the decoy ODN strategy. Specificity becomes an even greater challenge when target gene expression is to be inhibited only in a single organ or tissue type, since systemic delivery of the DNA is likely to lead to widespread uptake and potential nonspecific side effects. In addition, as with other ODN strategies, the successful use of transcription factor decoys will almost always depend on an efficient means to deliver the synthetic DNA to target cells. Although one recent report describes a mechanism by which decoy binding in the cytoplasm prevents nuclear translocation of NF- $\mathrm{KB}$ (31), for most applications, decoy ODNs are thought to require nuclear localization if they are to prevent the transactivation of their target genes. Unfortunately, the endocytotic pathways for phosphorothioate and other ODN uptake translocates most of the ODN into lysosomal compartments, where it is degraded.

A number of delivery vehicles have been explored to facilitate cellular uptake and nuclear localization of ODNs in intact tissues in vivo. Unfortunately, cationic lipids, which can greatly enhance plasmid DNA uptake, have only a modest impact on in vivo delivery of ODNs. The fusigenic hemagglutinating virus of Japan-liposome system has been used extensively for in vivo ODN delivery and appears to enhance nuclear localization (32, 33). More recently, our group has explored using controlled application of pressure to deliver functional ODNs to vascular tissues or solid organs $(34,35)$. This method can be used in either an ex vivo or an in situ approach $(34,36)$ and provides both tissue specificity and a high degree of safety via a highly localized delivery.

Although double-stranded DNA oligomers are the most widely studied of transcription factor decoy molecules, a number of groups are also pursuing structural modifications intended to enhance the stability and inhibitory effect of these agents. Several studies have examined cross-linking the two DNA molecules to form a single-stranded molecule folded on itself, either via photocrosslinking (37) or by the introduction of a covalently linked, nonnucleotide bridge (38). RNA decoy ODNs have been described that bind transcription factors via an aptameric interaction, as opposed to the naturally occurring sequence-directed binding site interaction (ref. 37; see also White et al. [ref. 39] and Hicke and Stevens [ref. 40], both in this Perspective series). In addition, circular decoys assuming a dumbbell configuration (41) or single-stranded decoys with a palindromic sequence that can fold on themselves (42) have also been described.

\section{Summary}

An improved understanding of the contributions of altered and pathologic gene expression to the pathogenesis of a wide variety of inherited and acquired diseases has paved a path for novel therapeutic strategies based on the manipulation of gene expression. The 
transcription factor decoy approach represents one such innovation that can allow researchers to effect gene activation or suppression in a specific fashion. The growing list of transcription factors linked to various types of cellular dysfunction defines a set of promising targets for this strategy, which is now gaining a firm foundation in cell culture and preclinical research. Early human clinical data with a decoy ODN targeting E2F provide proof of concept that human gene expression can be manipulated therapeutically. Ongoing investigations will undoubtedly provide a clearer picture of the roles these agents may play in molecular medicine.

\section{Acknowledgments}

This work was supported by grants HL-35610 and HL58516 from the NIH. V.J. Dzau is the recipient of a National Heart, Lung, and Blood Institute MERIT Award.

1. Bielinska, A., Shivdasani, R.A., Zhang, L.Q., and Nabel, G.J. 1990. Regulation of gene expression with double-stranded phosphorothioate oligonucleotides. Science. 250:997-1000.

2. Morishita, R., et al. 1995. A gene therapy strategy using a transcription factor decoy of the E2F binding site inhibits smooth muscle proliferation in vivo. Proc. Natl. Acad. Sci. USA. 92:5855-5859.

3. Gaubatz, S., Wood, J.G., and Livingston, D.M. 1998. Unusual proliferation arrest and transcriptional control properties of a newly discovered E2F family member, E2F-6. Proc. Natl. Acad. Sci. USA. 95:9190-9195.

4. Yamada, T., et al. 1995. In vivo identification of a negative regulatory element in the mouse renin gene using direct gene transfer. J. Clin. Invest. 96:1230-1237.

5. Tomita, S., et al. 1999. Transcription factor decoy to study the molecular mechanism of negative regulation of renin gene expression in the liver in vivo. Circ. Res. 84:1059-1066.

6. Cox, J.L., Chiasson, D.A., and Gotlieb, A.I. 1991. Stranger in a strange land: the pathogenesis of saphenous vein graft stenosis with emphasis on structural and functional differences between veins and arteries. Prog. Cardiovasc. Dis. 34:45-68.

7. Zwolak, R.M., Adams, M.C., and Clowes, A.W. 1987. Kinetics of vein graft hyperplasia: association with tangential stress. J. Vasc. Surg. 5:126-136. 8. Tanaka, H., et al. 1993. Sustained activation of vascular cells and leukocytes in the rabbit aorta after balloon injury. Circulation. 88:1788-1803.

9. Mann, M.J., Kernoff, R., and Dzau, V.J. 1997. Vein graft gene therapy using E2F decoy oligonucleotides: target gene inhibition in human veins and long term resistance to atherosclerosis in rabbits. Surgical Forum Volume. 48:242-244.

10. Mann, M.J., et al. 1997. Cell cycle inhibition preserves endothelial function in genetically engineered vein grafts. J. Clin. Invest. 99:1295-1301.

11. Mann, M.J., et al. 1999. Ex-vivo gene therapy of human vascular bypass grafts with E2F decoy: the PREVENT single-centre, randomised, controlled trial. Lancet. 354:1493-1498.

12. Idu, M.M., et al. 1993. Impact of a color-flow duplex surveillance program on infrainguinal vein graft patency: a five year experience. J. Vasc. Surg. 17:42-53.

13. Mercurio, F., and Manning, A.M. 1999. NF- $\mathrm{KB}$ as a primary regulator of the stress response. Oncogene. 18:6163-6171.

14. Collins, T., et al. 1995. Transcriptional regulation of endothelial cell adhesion molecules: NF- $\mathrm{KB}$ and cytokine-inducible enhancers. FASEB J. 9:899-909.

15. Khaled, A.R., Butfiloski, E.J., Sobel, E.S., and Schiffenbauer, J. 1998. Use of phosphorothioate-modified oligodeoxynucleotides to inhibit NF- $\kappa B$ expression and lymphocyte function. Clin. Immunol. Immunopathol. 86:170-179.

16. Griesenbach, U., et al. 2000. Anti-inflammatory gene therapy directed at the airway epithelium. Gene Ther. 7:306-313.

17. Tomita, T., et al. 1999. Suppressed severity of collagen-induced arthritis by in vivo transfection of nuclear factor $\mathrm{\kappa B}$ decoy oligodeoxynucleotides as a gene therapy. Arthritis Rheum. 42:2532-2542.

18. Kawamura, I., et al. 1999. Intratumoral injection of oligonucleotides to the NF $\kappa \mathrm{B}$ binding site inhibits cachexia in a mouse tumor model. Gene Ther. 6:91-97.

19. Tomita, N., et al. 1998. Transcription factor decoy for nuclear factor- $\kappa B$ inhibits tumor necrosis factor-alpha-induced expression of interleukin6 and intracellular adhesion molecule- 1 in endothelial cells. J. Hypertens. 16:993-1000.

20. Sawa, Y., et al. 1997. A novel strategy for myocardial protection using in vivo transfection of cis element 'decoy' against NFKB binding site: evidence for a role of NFKB in ischemia-reperfusion injury. Circulation. 96(Suppl. 9):II280-II284

21. Morishita, R., et al. 1997. In vivo transfection of cis element "decoy" against nuclear factor- $\mathrm{\kappa B}$ binding site prevents myocardial infarction. Nat. Med. 3:894-899.

22. Lim, R., Zaheer, A., Yorek, M.A., Darby, C.J., and Oberley, L.W. 2000. Activation of nuclear factor- $\mathrm{\kappa B}$ in $\mathrm{C} 6$ rat glioma cells after transfection with glia maturation factor. J. Neurochem. 74:596-602.

23. Takeuchi, S., et al. 1999. AP-2beta represses D(1A) dopamine receptor gene transcription in neuro2a cells. Brain Res. Mol. Brain Res. 74:208-216.

24. von Knethen, A., Brockhaus, F., Kleiter, I., and Brune, B. 1999. NO-Evoked macrophage apoptosis is attenuated by cAMP-induced gene expression. Mol. Med. 5:672-684.

25. Bishop-Bailey, D., and Hla, T. 1999. Endothelial cell apoptosis induced by the peroxisome proliferator-activated receptor (PPAR) ligand 15deoxy-Delta12, 14-prostaglandin J2. J. Biol. Chem. 274:17042-17048.

26. Boccaccio, C., et al. 1998. Induction of epithelial tubules by growth factor HGF depends on the STAT pathway. Nature. 39:285-288.

27. von Knethen, A., Lotero, A., and Brune, B. 1998. Etoposide and cisplatin induced apoptosis in activated RAW 264.7 macrophages is attenuated by cAMP-induced gene expression. Oncogene. 17:387-394.

28. Wang, L.H., Yang, X.Y., Kirken, R.A., Resau, J.H., and Farrar, W.L. 2000. Targeted disruption of stat 6 DNA binding activity by an oligonucleotide decoy blocks IL-4-driven T(H)2 cell response. Blood. 95:1249-1257.

29. Park, Y.G., Nesterova, M., Agrawal, S., and Cho-Chung, Y.S. 1999. Dual blockade of cyclic AMP response element- (CRE) and AP-1-directed transcription by CRE-transcription factor decoy oligonucleotide. Gene-specific inhibition of tumor growth. J. Biol. Chem. 274:1573-1580.

30. Lee, Y.N., Park, Y.G., Choi, Y.H., Cho, Y.S., and Cho-Chung, Y.S. 2000. CRE-Transcription factor decoy oligonucleotide inhibition of MCF-7 breast cancer cells: cross-talk with p53 signaling pathway. Biochemistry. 39:4863-4868.

31. Cooper, J.A., Jr., et al. 2000. Attenuation of interleukin-8 production by inhibiting nuclear factor- $\mathrm{\kappa B}$ translocation using decoy oligonucleotides. Biochem. Pharmacol. 59:605-613.

32. Morishita, R., et al. 1993. Single intraluminal delivery of antisense cdc2 kinase and proliferating-cell nuclear antigen oligonucleotides results in chronic inhibition of neointimal hyperplasia. Proc. Natl. Acad. Sci. USA. 90:8474-8478.

33. Dzau, V.J., Mann, M.J., Morishita, R., and Kaneda, Y. 1996. Fusigenic viral liposome for gene therapy in cardiovascular diseases. Proc. Natl. Acad. Sci. USA. 93:11421-11425.

34. Mann, M.J., et al. 1999. Pressure-mediated oligonucleotide transfection of rat and human cardiovascular tissues. Proc. Natl. Acad. Sci. USA. 96:6411-6416.

35. Poston, R.S., et al. 1999. Antisense oligodeoxynucleotides prevent acute cardiac allograft rejection via a novel, nontoxic, highly efficient transfection method. Transplantation. 68:825-832.

36. von der Leyen, H.E., et al. 1999. A pressure-mediated nonviral method for efficient arterial gene and oligonucleotide transfer. Hum. Gene Ther. 10:2355-2364.

37. Iwase, R., Namba, M., Yamaoka, T., and Murakami, A. 1997. Gene regulation by decoy approach (I): synthesis and properties of photocrosslinked oligonucleotides. Nucleic Acids Symp. Ser. 1997:203-204.

38. Amoah-Apraku, B., Fang, M.Z., and Guzman, N.J. 2000. A nonnucleotide-bridged DNA decoy inhibits renal epithelial nitric oxide synthase expression. Kidney Int. 57:83-91.

39. White, R.R., Sullenger, B.A., and Rusconi, C.P. 2000. Developing aptamers into therapeutics. J. Clin. Invest. 106:929-934.

40. Hicke, B.J., and Stephens, A.W. 2000. Escort aptamers: a delivery service for diagnosis and therapy. J. Clin. Invest. 106:923-928.

41. Lebruska, L.L., and Maher, L.J., III. 1999. Selection and characterization of an RNA decoy for transcription factor NF- $\kappa B$. Biochemistry. 38:3168-3174.

42. Hosoya, T., et al. 1999. Sequence-specific inhibition of a transcription factor by circular dumbbell DNA oligonucleotides. FEBS Lett. 461:136-140. 